Check for updates

Cite this: RSC Adv., 2017, 7, 36777

\title{
Integrated heart/cancer on a chip to reproduce the side effects of anti-cancer drugs in vitro $\uparrow$
}

\author{
Ken-ichiro Kamei, (D) *a Yoshiki Kato, ${ }^{b}$ Yoshikazu Hirai, ${ }^{\text {aab }}$ Shinji Ito, ${ }^{c}$ Junko Satoh, ${ }^{c}$ \\ Atsuko Oka, ${ }^{c}$ Toshiyuki Tsuchiya, ${ }^{b}{ }^{\text {b }}$ Yong Chen ${ }^{\text {ad }}$ and Osamu Tabata ${ }^{\mathrm{b}}$
}

Pre-clinical animal tests are used to assess drug efficacy and safety, but are limited by factors such as their suitability as a model for humans, robustness, cost, and ethical issues. While an organ-on-a-chip using human cells is promising for recapitulating human physiological conditions, it is highly desirable in investigations of the side effects of drugs to integrate more than one type of tissue using a designed circulatory system. We have developed a microfluidic device-an Integrated Heart/Cancer on a Chip (iHCC)-using human healthy heart cells (hCMs) and liver cancer cells (HepG2) to recapitulate the side effects of an anti-cancer drug, doxorubicin (DXR), to achieve individual cultures of cells from different tissues on a single device with three sets of artificial blood circulation loops, microfabrication technology for micro valves and a pump provides accurate fluid operation. Using improved soft lithography adopting numerical optimization simulation, the microfluidic device was fabricated with on-chip integration of pneumatic valves and a peristaltic micropump establishing precision fluid flow. The iHCC developed allows modelling of the side effects of DXR on heart cells caused by the production of toxic metabolites (doxorubicinol; DXRol) by HepG2 cells and the delivery of DXRol to heart cells via the circulation loop. Our findings open the door towards the development of a "Body-on-a-Chip."

Received 13th July 2017
Accepted 19th July 2017
DOI: 10.1039/c7ra07716e
rsc.li/rsc-advances
To address these issues, the "Organ on a Chip (OoC)"3-11 or "Body on a Chip (BoC)"12-19 technologies, which can mimic human physiological conditions in a microfluidic device, hold great promise as the next generation of pre-clinical tests. Indeed, OoC and BoC were considered as being the "Top 10 Emerging Technologies of 2016" by the World Economic Forum's Meta-Council on Emerging Technologies. In particular, BoC technology allows the establishment of in vitro pre-clinical drug tests capable of assessing absorption, distribution, metabolism, and excretion processes (ADME) for pharmaceutical compounds, all of which profoundly influence the performance and pharmacological activity of a compound. Because BoC consists of multiple tissues in a single device, it also allows observation of the interaction between different tissues.

However, the current BoC platforms have several issues that need to be solved prior to their realization. Most BoC platforms have either a one-way flow stream or a few branches on a chip, so they cannot completely mimic the blood circulation system, or perform ADME tests for periods of days or weeks. Although Maschmeyer et al. reported a four-organ-chip enabling coculture of human intestine, liver, skin, and kidney cells with a medium circulation loop, ${ }^{20,21}$ it has not been using for drug testing, and is still at the initial stage. Some studies have achieved medium circulation using an external rotary peristaltic pump, but this requires a large amount of culture medium, and drugs and metabolites might be lost to the tubing. ${ }^{22,23}$ To the best of our knowledge, current devices have either a closed

$\dagger$ Electronic supplementary information (ESI) available. See DOI: 10.1039/c7ra07716e 
circulation loop to interconnect tissues or multiplexed capability within a single device. It is important to note that most of the aforementioned OoC or BoC systems have only a single set of experimental conditions in a chip, and do not allow side-byside comparisons within a device. Furthermore, the direct integration of micropumps in the device often causes difficulties in processing, such as incompatibility and reproducibility, because the existing fabrication methods ${ }^{24-28}$ are not simple and reliable. Therefore, it is important to develop new microfabrication technologies capable of integrating pumps into a device to minimize the volume of the tested medium and prevent sample loss.

Here, we introduce a BoC, which we have named the Integrated Heart/Cancer on a Chip (iHCC), which offers (1) culture of cells of different tissues within a single device, (2) an artificial blood circulatory system in the form of a closed circulation loop without the use of an external pump, and (3) three sets of loops that can be used to apply different conditions. By using this iHCC, we provide proof of principle for recapitulation of the side effects and cardiotoxicity of an anti-cancer drug in vitro (Fig. 1). The cardiotoxicity induced by anti-cancer drugs ${ }^{29}$ is a major and serious concern in drug discovery, and the drug metabolites generated from cancerous tissues might exert such side effects on normal tissues via the blood circulatory system (Fig. 1A). In this study, doxorubicin (DXR) ${ }^{30}$ was selected (Fig. 1B) as a model anti-cancer drug. DXR was originally obtained from Streptomyces peucetius var. caesius, acts through DNA intercalation, and has been used for treating a variety of tumors, such as those of the lung, bladder, liver, breast, and prostate. ${ }^{31}$ DXR exerts serious side effects on the heart, such as cardiomyopathy caused by undesired apoptosis. ${ }^{32}$ The use of

A

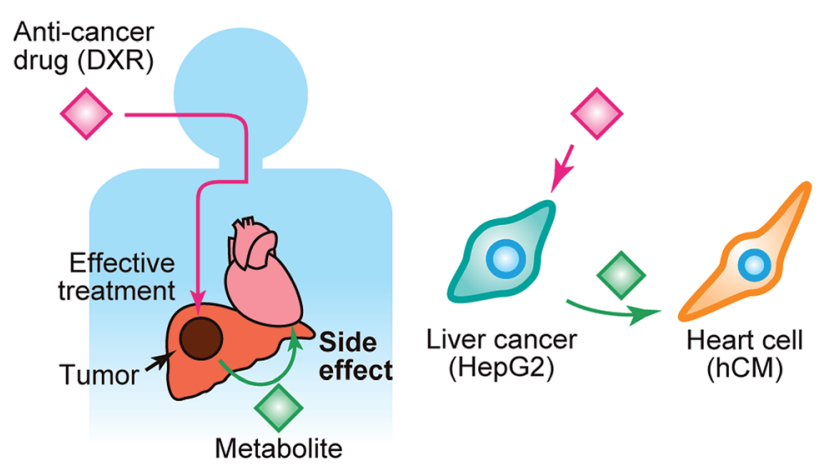

Fig. 1 Communication between liver cancer cells and healthy heart cells via exchange of metabolites, and the side effects of an anticancer drug. (A) Illustration of the anti-cancer effect of doxorubicin (DXR) according to the metabolic activity of liver cancer and the side effects of a DRX metabolites on the healthy heart. Although the anticancer drug could effectively cure liver cancer, its metabolite might travel to healthy tissues, such as the heart, and cause damage to those tissues. (B) Our proposed in vitro model to recapitulate the side effects of DXR using two types of cells: a liver cancer cell line (HepG2) and primary human cardiomyocytes (hCM). In the co-culture system, a drug metabolite might be released from HepG2 cells by metabolic activity, and reach hCM cells, thereby causing cell damage. this combination of cells enables modelling of damage to the heart caused by both the anti-cancer drug and its metabolite produced in cancer cells. In this study, we attempted to reproduce the side effects of DXR using human primary cardiomyocytes (hCMs) from a healthy donor and human hepatocellular carcinoma HepG2 cells, which have been widely used as a cancer cell model for the screening of anti-cancer drugs (Fig. 1B). ${ }^{22,23}$

\section{Experimental}

\section{Materials and methods}

Design of the microfluidic device used for iHCC. The iHCC was constructed from polydimethylsiloxane (PDMS; SYLGARD 184, Dow Corning), consisting of a perfusion layer and a control layer. PDMS was selected because it has good biocompatibility, gas permeability, and light transparency (Fig. 2A). ${ }^{33,34}$ The perfusion layer contained two cell culture chambers $(2.1 \mathrm{~mm}$ in width and $220 \mu \mathrm{m}$ in height), sufficient to supply the required growth factors, the anti-cancer drug, and allowing for reduced shear stress. ${ }^{35}$ Microchannels were used to interconnect the two cell culture chambers $(150 \mu \mathrm{m}$ in width, $45 \mu \mathrm{m}$ in height) through inlet and outlet holes. The control layer, consisting of a thin and flexible PDMS membrane $\left(200 \times 200 \mu \mathrm{m}^{2}\right.$, and $20 \mu \mathrm{m}$ thickness), contained pneumatic valves ${ }^{24}$ to allow for the independent culture of two types of cells without cross contamination, and peristaltic micro pumps ${ }^{36}$ for precision medium flow control. Membrane actuation at the valves and pumps was performed by varying the positive hydraulic pressure from the control channel located under the perfusion layer, which was achieved using computer-controlled solenoid valves for accurate control. The micro pump consisted of three individual valves, providing a peristaltic motion for programmable fluid actuation. This micro pump was utilized for an embedded closed-circulation loop ${ }^{35}$ as an artificial circulation system to interconnect different cells (Fig. 2B). The set of micropumps and embedded circulation loop minimizes the loss of medium volume and reduces sample loss. Thus, this device comprised three sets of two cell culture chambers with a closed-circulation loop to allow for three different but simultaneous cell treatments within a device (Fig. 2C).

Device fabrication. A multilayer soft lithography replica molding technique was used for device fabrication (Fig. 3). ${ }^{24,37}$ The control layer was cast against the negative photoresist mold with microfluidic channel features with a thickness of $30 \mu \mathrm{m}$ (TMMR S2000, Tokyo Ohka Kogyo) patterned by standard UV lithography. A resist mold for the perfusion layer was fabricated by a combination of standard UV lithography and grayscale lithography. First, the negative resist layer (TMMF S2045, Tokyo Ohka Kogyo) (thickness, $220 \mu \mathrm{m}$ ) was patterned for the perfusion layer using standard UV lithography. Next, the positive resist layer (PMER P-LA900PM, Tokyo Ohka Kogyo) was spincoated at a thickness of $45 \mu \mathrm{m}$ to fabricate the resist mold for the microchannels and pneumatic portions, and then digital micromirror device (DMD)-based lithography (DL-1000GS/KCH, NanoSystems Solutions) was performed. ${ }^{38}$ 
A

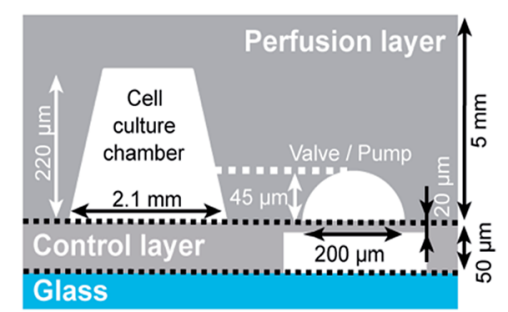

B
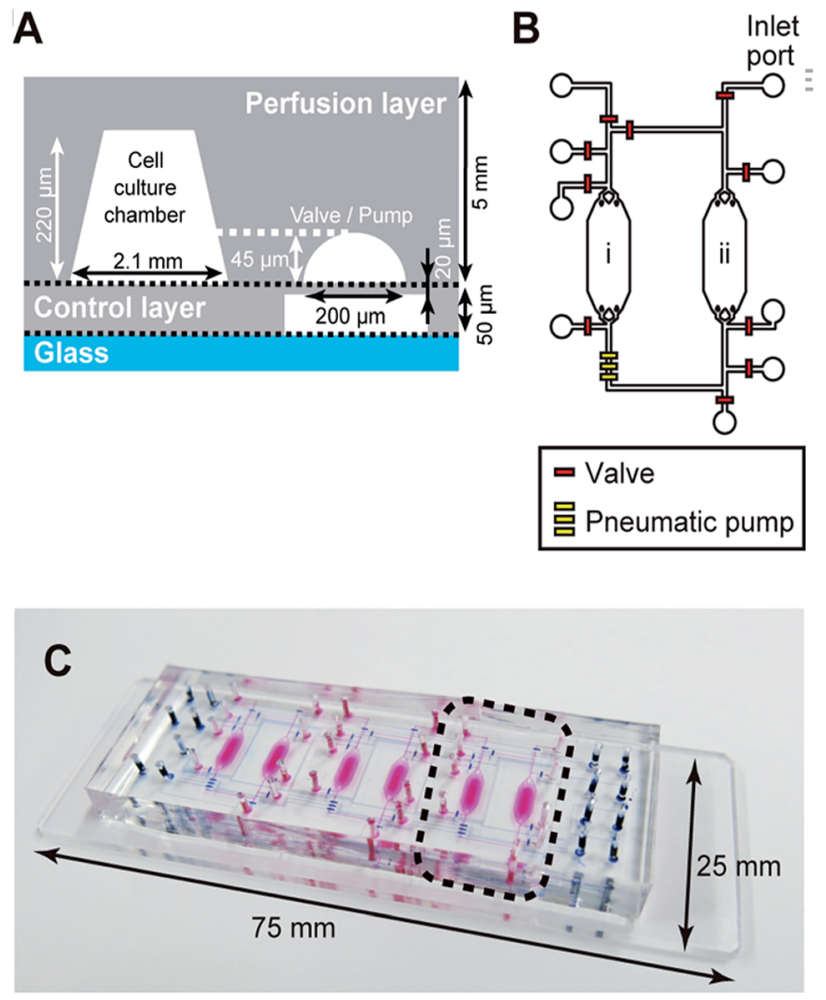

Fig. 2 Design of the microfluidic device used for iHCC. (A) Illustration of the side view of iHCC. The device consists of a "perfusion layer" and "control layer", and was constructed from polydimethylsiloxane (PDMS) as shown in grey. (B) A set of cell culture chambers with a medium circulation system mimics the blood circulatory system that interconnects various tissue cells. Integrated valves (red) allow precise control of the fluids as well as introduction of cell/reagents. A pneumatic pump (yellow) provides medium circulation within a set of two cell culture chambers ("i" and "ii" for HepG2 and hCM, respectively). (C) Photograph of an actual iHCC fabricated on a glass slide $(25 \mathrm{~mm} \times 75$ $\mathrm{mm})$. This device has three sets of co-culture systems, and the dashed line indicates one of them as shown in (B). The operation of pneumatic valves and peristaltic pumps is controlled by custom LabVIEW-based software. The pink color indicates the top layer containing the cell culture chambers, and the dark blue color indicates the bottom layer containing the valves and pumps.

For the pneumatic portion, DMD-based grayscale lithography using numerical optimization ${ }^{38,39}$ was employed for wafer-level fabrication (Fig. 3).

After fabrication of the resist molds, the PDMS base and curing agents (1:10 with a weight ratio, curing agent : base) were mixed well, degassed in a vacuum desiccator, and poured on the mold structures for the perfusion layer. After curing in a convection oven at $80{ }^{\circ} \mathrm{C}$, the perfusion layer was released from the resist mold. Similarly, for the PDMS layer (thickness, $20 \mu \mathrm{m})$, the PDMS membrane was fabricated with the control layer by spin-coating the PDMS pre-polymer onto a resist mold. After spin-coating, the control layer was cured at $80{ }^{\circ} \mathrm{C}$. The two cured PDMS layers were aligned under a microscope and bonded onto the control layer using the partial PDMS curing method, ${ }^{\mathbf{4 0 4 1}}$ allowing for the spontaneous adhesive interaction between the two PDMS layers. Finally, the assembled device was permanently bonded using $\mathrm{O}_{2}$ plasma surface treatment (FA-1, SAMCO) onto a microscopic glass slide $(25 \mathrm{~mm} \times 75 \mathrm{~mm})$.

Device control. The pneumatic control setup consisted of two sets of eight-channel manifolds (Microfluidic System Works Inc. and THE LEE Company). The setup was controlled using a controller board (VC3 8 controller [ALA Scientific Instruments] and NI USB-6501 [National Instruments]), connected to a computer through a USB port. Compressed nitrogen gas provided pressure $(200 \mathrm{kPa})$ to the manifolds. Metal pins containing Tygon Microbore tubing (Saint-Gobain) filled with water were inserted to the holes of the top perfusion layer connected to the valves and pump, as indicated by the dark blue color shown in Fig. 2C. The other end of the Tygon Microbore tubing was connected to the corresponding control channels on the manifolds. Control channels in the chips were first filled with DI-water by applying pressure $(30 \mathrm{kPa})$. The electronic manifold interface was created using LabVIEW (Version 11.0, National Instrument Inc.).

Cell culture. HepG2 hepatocellular carcinoma cells were obtained from American Type Culture Collection. HepG2 cells were maintained in Dulbecco's modified Eagle medium (DMEM; Thermo Fisher Scientific) supplemented with 10\% (v/v) fetal bovine serum (FBS, Cell Culture Bioscience), 1\% (v/v) nonessential amino acids (Thermo Fisher Scientific), and 1\% (v/v) penicillin/streptomycin (Thermo Fisher Scientific), referred to as HepG2 medium. Culture medium was changed every two days, and the cells were passaged with TrypLE express every four days.

Human primary cardiomyocytes (hCMs) were obtained from PromoCell, and were maintained in myocyte medium (PromoCell) supplemented with $0.05 \%(\mathrm{v} / \mathrm{v})$ fetal calf serum (PromoCell), $0.5 \mathrm{ng} \mathrm{mL}^{-1}$ recombinant human epidermal growth factor (PromoCell), $2 \mathrm{ng} \mathrm{mL}^{-1}$ recombinant human basic fibroblast growth factor (PromoCell), and $5 \mu \mathrm{g} \mathrm{mL}^{-1}$ recombinant human insulin (PromoCell) in a humidified incubator at $37{ }^{\circ} \mathrm{C}$ and $5 \%$ (v/v) $\mathrm{CO}_{2}$. Cell culture medium was changed every three days, and the cells were passaged using a trypsin/ ethylenediaminetetraacetic acid (EDTA) $(0.04 \% / 0.03 \%[\mathrm{v} / \mathrm{v}])$ solution (Thermo Fisher Scientific) every week.

Cell culture in iHCC. Prior to the use of iHCC for cell culture, the chip was washed with $70 \%(\mathrm{v} / \mathrm{v})$ ethanol and irradiated with UV light to prevent contamination. Next, $100 \mu \mathrm{g} \mathrm{mL}{ }^{-1}$ human recombinant fibronectin (Thermo Fisher Scientific), $0.1 \%(\mathrm{w} / \mathrm{v})$ bovine gelatin (Type B, Sigma-Aldrich), or $1.3 \%(\mathrm{v} / \mathrm{v})$ Matrigel hESC (human embryonic stem cell)-Qualified Matrix (Corning) in DMEM/F12 medium (Thermo Fisher Scientific) was introduced into the chip using a syringe inserted at the inlet hole, and incubated at $4{ }^{\circ} \mathrm{C}$ for at least 16 hours to allow for coating of the extracellular matrices. After rinsing excess Matrigel with DMEM/F12 medium, the chip was placed in an incubator at $37{ }^{\circ} \mathrm{C}$ until use.

HepG2 and hCM cells were harvested from culture flasks using $2 \mathrm{~mL}$ of trypsin/EDTA $(0.04 \% / 0.03 \%$ [v/v]) solution and incubated at $37{ }^{\circ} \mathrm{C}$ for 5 minutes. After addition of $1 \mathrm{~mL}$ of trypsin neutralizing solution (TNS; PromoCell) to the hCM sample, HepG2 and hCM cells were resuspended in their corresponding medium at $1.0 \times 10^{6}$ cells per mL. Following this, 


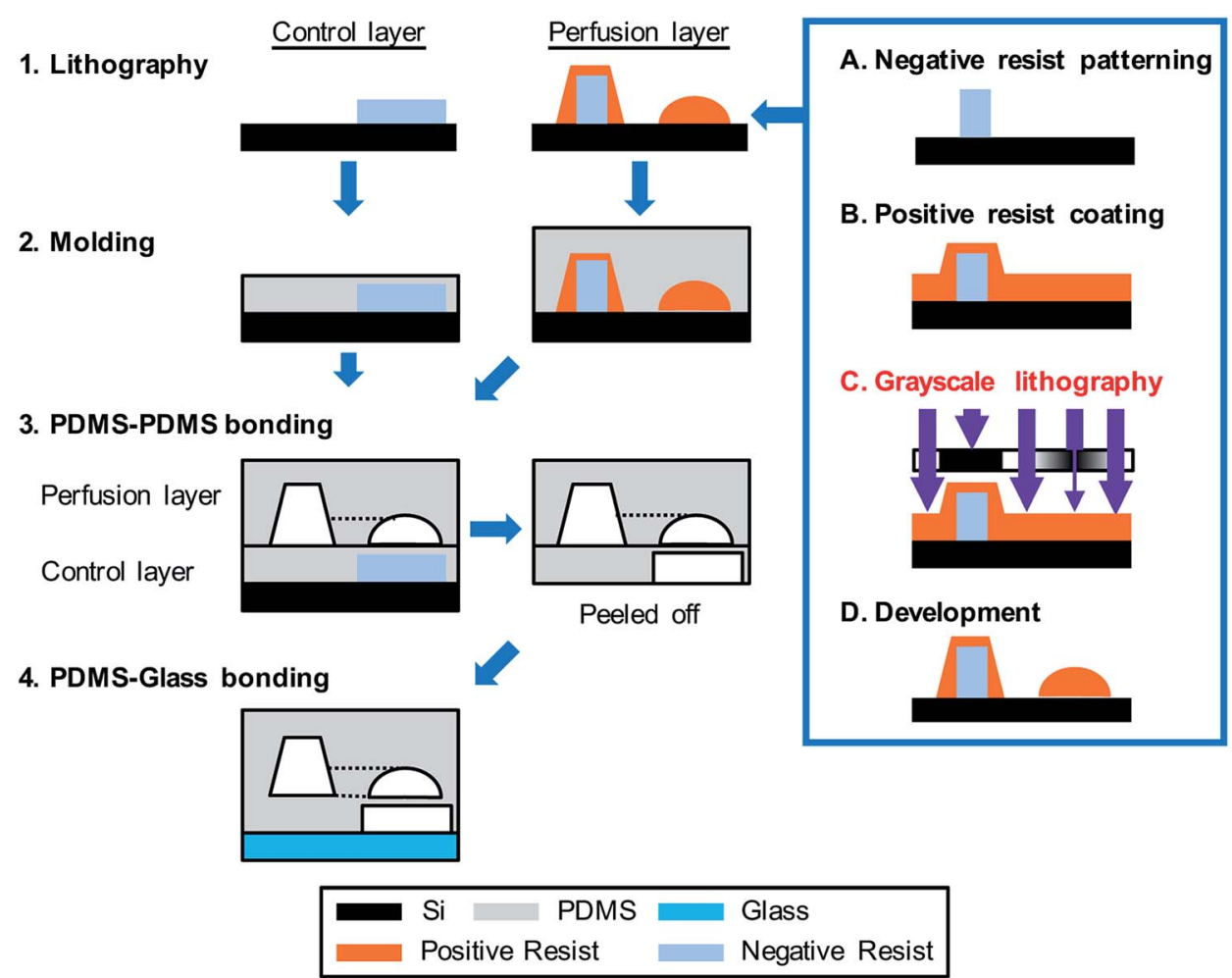

Fig. 3 Fabrication of the integrated Heart/Cancer on a Chip (iHCC) microfluidic device. The device was fabricated as follows: (1) for the control layer, the negative resist was patterned on a Si wafer and was used as a casting mold. A casting mold of the perfusion layer was fabricated by a combination of the negative resist patterning and grayscale lithography using positive resist coating. Following this, digital micromirror device (DMD)-based grayscale lithography (DL-1000GS/KCH, NanoSystems Solutions) combined with the simulation-based fabrication approach was employed as described in the previous reports. ${ }^{38,39}$ (2) The PDMS pre-polymer was poured onto the mold structure and cured. (3) The perfusion layer was bonded onto the control layer. (4) The assembled structure containing the perfusion and control layers was bonded onto a glass substrate using oxygen plasma treatment.

$10 \mu \mathrm{L}$ of the cell suspension was introduced using a pipette into the hole adjacent to the cell culture chamber to plate the device with cells at $7.0 \times 10^{4}$ cells per $\mathrm{cm}$. The cell-loaded device was then placed in a humidified incubator at $37{ }^{\circ} \mathrm{C}$ under $5 \%(\mathrm{v} / \mathrm{v})$ $\mathrm{CO}_{2}$.

Cell treatment in iHCC. Prior to cell treatment with doxorubicin (DXR; Sigma), DXR was dissolved in dimethylsulfoxide (DMSO; Wako), to give a $10 \mathrm{mM}$ DXR stock solution. Cells were treated with $20 \mu \mathrm{M}$ DXR in $0.1 \%$ (v/v) DMSO in hCM medium for 24 hours with or without medium circulation. As positive and negative controls (PC and NC, respectively) for cell damage, hCM medium supplemented with $1 \mu \mathrm{M}$ staurosporine (STS; Wako) in $0.1 \%$ (v/v) DMSO, or $0.1 \%$ (v/v) DMSO, were used respectively. Medium circulation was applied using an integrated pump at $0.25 \mathrm{~Hz}$ and $200 \mathrm{kPa}$, controlled by the LabVIEW program (National Instruments). Every six hours, fresh hCM medium, present in the tubing inserted at the inlet port, was introduced automatically. During cell treatment, the iHCC was placed in a humidified incubator at $37{ }^{\circ} \mathrm{C}$ under $5 \%(\mathrm{v} / \mathrm{v}) \mathrm{CO}_{2}$.

Cell treatment in a 96-well plate. Following the same procedure that was used in "Cell culture in iHCC," HepG2 and hCM cells were harvested from a cell culture flask. Subsequently, $100 \mu \mathrm{L}$ of $1 \times 10^{5}$ cells per mL cell suspension in their corresponding medium was introduced in each well of a 96-well plate to give a cell density of $7.0 \times 10^{4}$ cells per $\mathrm{cm}$, and then cultured in a humidified incubator at $37{ }^{\circ} \mathrm{C}$ under $5 \%(\mathrm{v} / \mathrm{v}) \mathrm{CO}_{2}$ for 24 hours. For cell treatment, $20 \mu \mathrm{M}$ DXR in hCM medium containing $0.1 \%(\mathrm{v} / \mathrm{v})$ DMSO was used. As PC and NC for cell damage, hCM medium supplemented with $1 \mu \mathrm{M}$ STS in $0.1 \%$ (v/ v) DMSO or with only $0.1 \%(\mathrm{v} / \mathrm{v})$ DMSO, respectively, was used.

Staining of proliferating cells. Staining of proliferating cells with EdU was performed according to the product manuals of the Click-iT EdU Imaging Kit (Thermo Fisher Scientific). Briefly, after cell treatments, cells were treated with hCM medium supplemented with $10 \mu \mathrm{M}$ EdU in a humidified incubator at $37{ }^{\circ} \mathrm{C}$ under $5 \%(\mathrm{v} / \mathrm{v}) \mathrm{CO}_{2}$ for $30 \mathrm{~min}$. After washing away excess EdU with hCM medium, the cells were fixed with $4 \%$ paraformaldehyde for $15 \mathrm{~min}$ at $25{ }^{\circ} \mathrm{C}$, followed by permeabilization (0.3\% Triton X-100 in PBS) for 16 hours at $25{ }^{\circ} \mathrm{C}$. EdU incorporated into cellular DNA was labeled with Alexa Fluor 647 by means of a click reaction.

Dead cell staining. After cell treatment, cells were treated with $300 \mathrm{nM} \mathrm{4}$,6-diamidino-2-phenylindole (DAPI, Thermo Fisher Scientific) dissolved in hCM medium (without DXR, STS, or DMSO), and incubated in a humidified incubator at $37{ }^{\circ} \mathrm{C}$ under $5 \%(\mathrm{v} / \mathrm{v}) \mathrm{CO}_{2}$ for $30 \mathrm{~min}$. Next, excess fluorescent dye was rinsed off and replaced with DMEM/F12 medium without phenol red for cell microscopic imaging. 
Image acquisition and processing. The device was placed on the stage of a Nikon ECLIPSE Ti inverted fluorescence microscope equipped with a CFI Plan Fluor $4 \times / 0.13$ N.A. objective lens (Nikon), CCD camera (ORCA-R2, Hamamatsu Photonics), mercury lamp (Intensilight, Nikon), XYZ automated stage (Ti-SER motorized stage with encoders, Nikon), and filter cubes for two fluorescence channels (DAPI and Cy5; Nikon). The images were acquired using all motorized components (stage, filters, shutter, and focus system) that were controlled by the manufacturer's NIS-elements imaging software. The exposure times were set at $1 \mathrm{~s}$ for DAPI (DAPI channel) and EdU (Cy5 channel). Following image acquisition, the CellProfiler software (Broad Institute of Harvard and MIT, Version 2.1.0 $)^{\mathbf{4 2}}$ was used for identifying cells by employing Otsu's method, and the fluorescent signals in individual cells were quantified automatically.

Ultra-high-performance liquid chromatography-tandem mass spectrometry. Culture medium $(24 \mu \mathrm{L})$ was mixed with 1 $\mu \mathrm{L}$ of internal standard (IS) solution. IS solution was prepared by mixing daunorubicin (DNM) hydrochloride (Sigma) with fresh culture medium at $3 \mu \mathrm{M}$. The solution was mixed with 75 $\mu \mathrm{L}$ of acetonitrile, vigorously mixed for $30 \mathrm{~s}$, and centrifuged $\left(16000 \times g, 10 \mathrm{~min}, 4{ }^{\circ} \mathrm{C}\right)$, and $30 \mu \mathrm{L}$ of the supernatant was mixed with $82.5 \mu \mathrm{L}$ of $5.45 \mathrm{mM}$ ammonium acetate ( $\mathrm{pH} 3.5)$. Part of the resulting solution $(30 \mu \mathrm{L})$ was separated on a Nexera UHPLC system (Shimadzu) using a binary gradient. The gradient program was as follows: 20 to $30 \%(\mathrm{v} / \mathrm{v}) \mathrm{B}$ in $8 \mathrm{~min}, 40$ to $100 \%(\mathrm{v} / \mathrm{v}) \mathrm{B}$ in $2 \mathrm{~min}, 100 \%(\mathrm{v} / \mathrm{v}) \mathrm{B}$ for $2 \mathrm{~min}, 100$ to $20 \%(\mathrm{v} / \mathrm{v})$ $\mathrm{B}$ in $0.01 \mathrm{~min}$, then $20 \%(\mathrm{v} / \mathrm{v}) \mathrm{B}$ for $3 \mathrm{~min}$. The flow rate was set to $0.4 \mathrm{~mL} \mathrm{~min}^{-1}$. Mobile phases $\mathrm{A}$ and B were $5 \mathrm{mM}$ ammonium acetate $(\mathrm{pH} 3.5)$ and acetonitrile, respectively. An ACQUITY UPLC CSH C18 column (130 ̊, $1.7 \mu \mathrm{m}, 2.1 \mathrm{~mm} \times 100 \mathrm{~mm}$ (Waters)) was used at $40{ }^{\circ} \mathrm{C}$. The UHPLC eluates were infused online to the LC-MS 8030 plus system (Shimadzu), which was set to positive electrospray ionization (ESI) mode. The responses for DXRol, DXR, and DNM were monitored by multiple reaction monitoring (MRM) with transitions $m / z 546.10>399.20,544.10$ $>397.20$, and $528.15>321.15$, respectively. All measurements were obtained in triplicate and the averaged responses were used. MRM transitions were optimized and peak areas were calculated using Labsolutions software (Shimadzu). The responses for DXRol and DXR were normalized to that of DNM in each sample. The calibration plots were prepared by measuring standard samples, which were generated by adding increasing amounts of DXR and DXRol and fixed amounts of DNM to fresh medium. Because HepG2 cells and hCMs were cultured with different media, the calibration plots were independently prepared for each medium to compensate for matrix effects.

Lactate dehydrogenase (LDH) cytotoxicity assay. The LDH cytotoxicity assay (Cytotoxicity Detection Kit PLUS, Roche) was performed by following the manufacturer's instruction. Briefly, after cell stimulation, the cell culture supernatant was harvested, and $100 \mu \mathrm{L}$ of the supernatant was transferred into a well of a fresh 96-well plate. Then, $100 \mu \mathrm{L}$ of a reaction mixture in the kit was added to the well and incubated at room temperature for $10 \mathrm{~min}$. The reaction was stopped by adding the stop solution in the kit. Finally, the absorbance of the samples was measured at 490 and $690 \mathrm{~nm}$.

Statistics. Statistical analyses, such as Mann Whitney's $U$ test and the Steel-Dwass test, were performed using R software (ver 3.2.2; https://www.r-project.org//).

\section{Results and discussion}

\section{Device characterization}

To characterize the performance of the directly embedded valves and the micropump, the threshold hydraulic pressure to completely close the microchannels was calibrated before the cell culture experiments. During the calibration, the control channels in the control layer were filled with DI water and pressurized using nitrogen gas with a pressure regulator. The inlet and outlet of the perfusion layer were connected to tubes
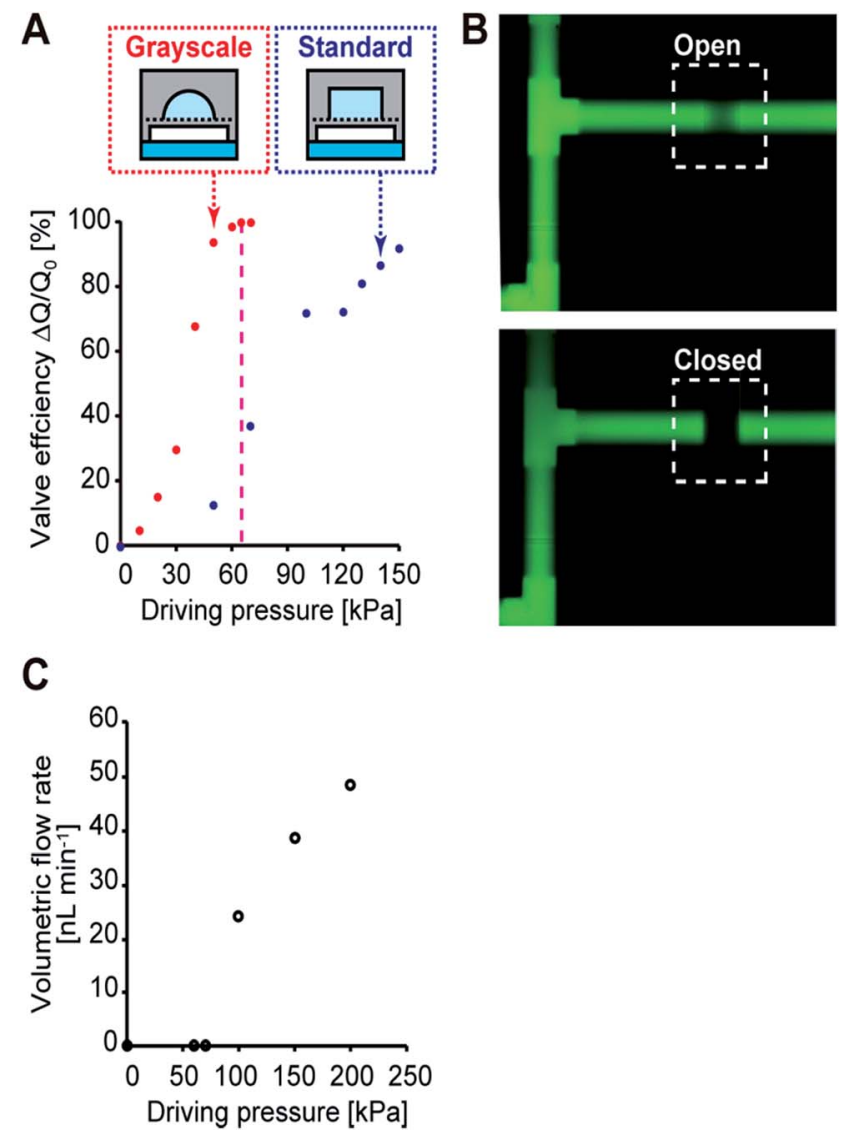

Fig. 4 Characterization of the embedded micro valves and pump. (A) A valve efficiency $(\Delta Q / Q)$ comparison between grayscale lithography and standard lithography. $\Delta Q$ and $Q$ are the flow rate change and flow rate when the valve is opened, respectively. In the case of standard lithography, a square shaped microchannel was used, which has the same width and height as that fabricated using grayscale lithography. (B) Fluorescent images of the integrated pneumatic valve in the open and closed states at the applied hydraulic pressure of $65 \mathrm{kPa}$. (C) Volumetric flow rate generated by the micro pump. The flow was measured by observing the moving water front without gravity flow. The measured volumetric flow rate was observed after applying

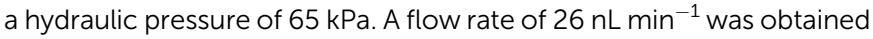
under a driving pressure of $100 \mathrm{kPa}$ at $2 \mathrm{~Hz}$ at the micropump. 
A

(1) Matrigel coating Matrigel

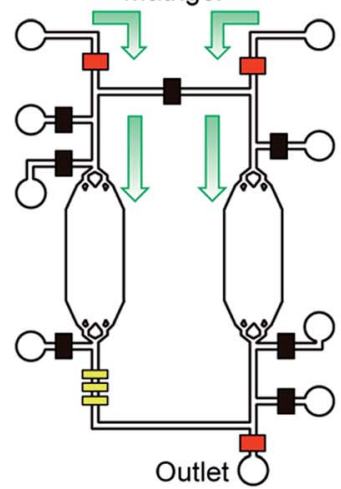

(2) Cell introduction

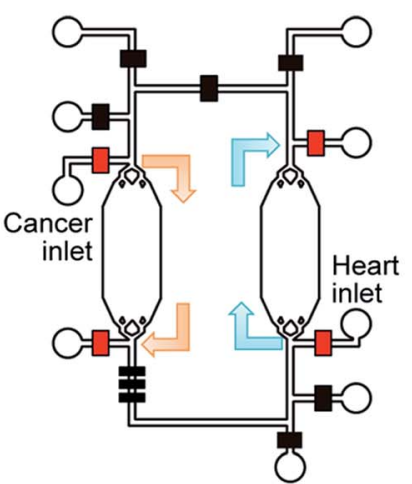

(3) Circulation

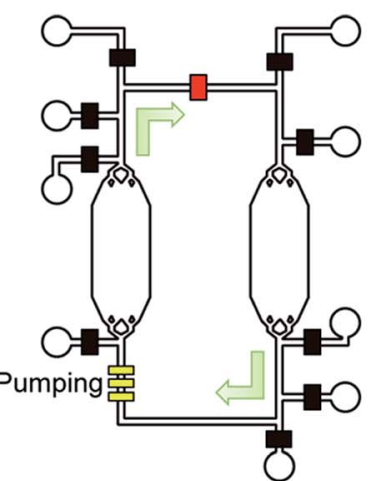

(4) Cell staining

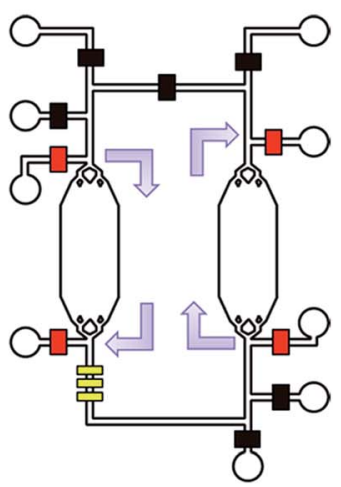

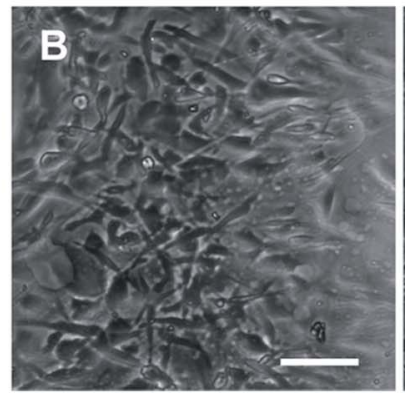

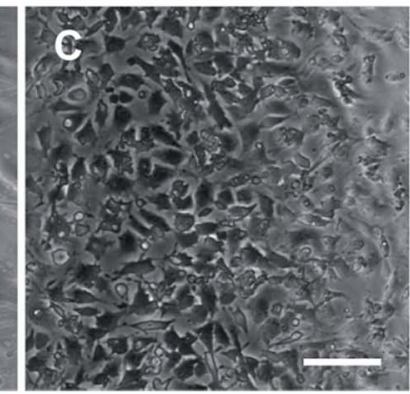

Fig. 5 Cell culture in the iHCC. (A) Procedure for cell culture and drug testing in the iHCC. The red and black squares represent the open and closed valves, respectively. The set of three yellow squares represents a peristaltic pump comprised of three pneumatic valves. (1) To facilitate cell adhesion and growth, Matrigel was first introduced using a syringe inserted at the inlet hole, and coated in the cell culture chamber at $4{ }^{\circ} \mathrm{C}$ for at least 16 hours after which the excess Matrigel was removed. (2) Following this, both cancer and heart cells were introduced into separate chambers using a pipette and a hole adjacent to the cell culture chamber, and incubated in a humidified incubator at $37^{\circ} \mathrm{C}$ for 24 hours. (3) After cell culture, medium circulation was started, with and without the drug solution, using the embedded micropumps for 24 hours. (4) Finally, DAPI was introduced and cells were incubated in a humidified incubator at $37^{\circ} \mathrm{C}$ for 30 min to observe cell damage. See also ESI $\dagger$ (B, C) microphotograph of human primary cardiomyocytes (hCMs; B) and HepG2 cells (C) cultured in iHCC under medium circulation after 24 hours. Scale bar, $100 \mu \mathrm{m}$.

and the gravity flow of DI water was generated at a pressure head of $400 \mathrm{~mm}$. The flow rate was measured by means of the progress of the water front in the tube, and the valve efficiency was then defined by a change in relative flow rate. The efficiency of the valve fabricated by grayscale lithography increased steeply until it reached hydraulic pressure of $63 \mathrm{kPa}$ compared to that of standard lithography (Fig. 4A). Taken together with the numerical simulation of a $200 \times 200 \mu \mathrm{m}$ square PDMS membrane having a threshold hydraulic pressure of $65 \mathrm{kPa}$ (CoventorWare, Coventor), the measurements agree with the threshold hydraulic pressure obtained from the simulation (differing by less than $3 \%$ ). ${ }^{39}$ The fluorescent images of the integrated pneumatic valve in the open and closed states, revealed that the valve was $100 \%$ closed at a hydraulic pressure of $65 \mathrm{kPa}$ (Fig. 4B). On the other hand, the efficiency of the valve fabricated by standard lithography continuously increased and did not close completely due to restrictions in PDMS's mechanical deformability at the microchannel edges. The volumetric flow rate generated by the micropump was also measured by observing the moving water front without gravity flow (Fig. 4C). The measured volumetric flow rate was observed after the applied hydraulic pressure reached $65 \mathrm{kPa}$; under a driving pressure of $100 \mathrm{kPa}$, a flow rate of $26 \mathrm{~nL} \mathrm{~min}{ }^{-1}$ was obtained at $2 \mathrm{~Hz}$. At this flow rate, drugs and metabolites moved from one chamber to another in two minutes. These data show that the present microfabrication technology can achieve reliability and accurate valve and micro pump actuation.

\section{Cell culture in iHCC}

To accomplish our iHCC goal, we first confirmed the applicability of our device for co-culturing two different cell types with circulation of the medium and then established an in vitro cellbased assay for reproduction of the side effects of DXR in the device.

To determine whether the fabricated device allowed simultaneous culture of two different cells, we introduced hCMs and HepG2 cells into the device and cultured them (Fig. 5A). Prior to cell culture, the culture chambers were coated with extracellular matrix (ECM) components such as fibronectin, gelatin, or 
A
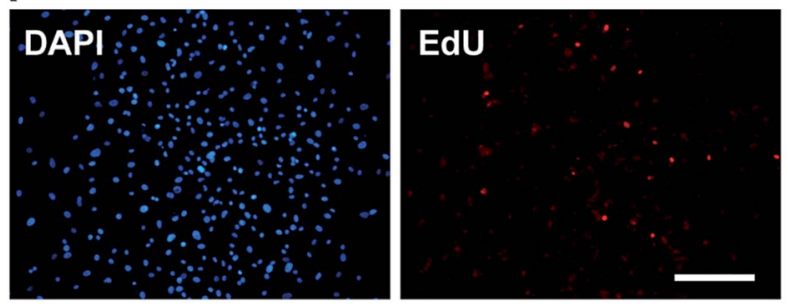

B
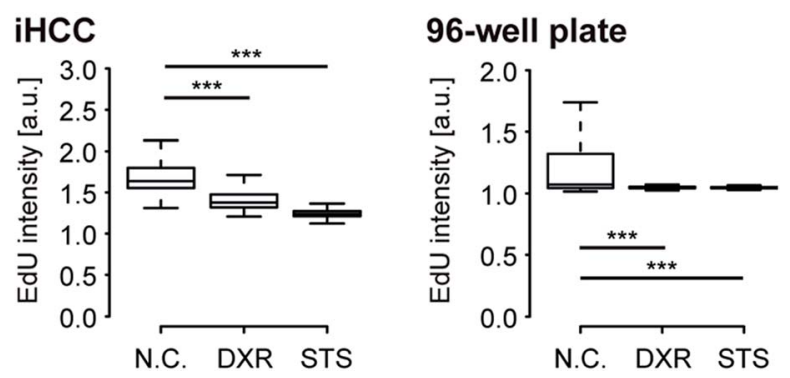

Fig. 6 Doxorubicin (DXR) has anti-cancer effects against HepG2 hepatocellular carcinoma cells by reducing the number of proliferating cells in both the iHCC and in a 96-well plate. (A) Fluorescent micrographs of fixed HepG2 cells stained with 300 nM DAPI and $10 \mu \mathrm{M}$ EdU in the iHCC. HepG2 cells were cultured in hCM medium in the iHCC for 24 hours. EdU stains only proliferating cells. DAPI was used to stain all nuclei in the fixed cells. Scale bar, $200 \mu \mathrm{m}$. (B) Quantitative singlecell profiling to reveal the effects of $20 \mu \mathrm{M}$ DXR in hCM medium for 24 hours on living HepG2 cells cultured using the iHCC (left) and a 96well plate (right). Fluorescent EdU intensity in over 3000 cells was analysed for each sample. Cell treatment with $1 \mu \mathrm{M}$ staurosporine (STS) for 24 hours was used as a positive control for cell damage. hCMs treated with only $0.1 \%$ DMSO in hCM medium for 24 hours were used as a negative control (N.C.). DAPI staining was performed to visualize damaged cells. P-Values $(* * *<0.001)$ were determined by Mann Whitney's $U$ test. The center lines show the medians; box limits indicate $25 \%$ and $75 \%$; whiskers extend 1.5 times the interquartile range from $25 \%$ and $75 \%$.

Matrigel at $4{ }^{\circ} \mathrm{C}$ for at least 16 hours. Matrigel was found to be a more effective ECM than fibronectin and gelatin for both cell types, in terms of cell proliferation for the first 24 hours (data not shown). When changing the medium, $10 \mu \mathrm{L}$ of fresh culture medium for each cell type was placed in a tube and introduced from an inlet into the respective cell culture chamber using a pipette. During cell culture, iHCC was placed in a humidified incubator at $37{ }^{\circ} \mathrm{C}$ under $5 \%(\mathrm{v} / \mathrm{v}) \mathrm{CO}_{2}$. After introducing the cells, it took approximately three hours for the cells to attach to the Matrigel-coated substrate. Because PDMS is a porous material, $\mathrm{CO}_{2}$ was able to penetrate through the PDMS of iHCC and reach the cell culture chambers. Therefore, the $\mathrm{pH}$ in iHCC was maintained due to the equilibrium of $\mathrm{HCO}_{3}{ }^{-}$and $\mathrm{CO}_{2}$. Both cell types reached over $80 \%$ confluence after one day of culture on the chip without any damage. After establishing cell culture in each chamber, we also set up a closed-loop medium circulation. For this, we used hCM medium, which was changed every 6 hours. Although hCM medium was not established for HepG2 cells, both HepG2 and hCM cells showed normal morphology as well as growth in the circulating medium
A

N.C.

DXR STS
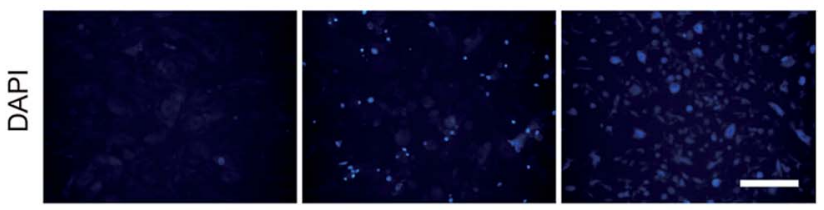

B 96-well plate (Static condition) HepG2
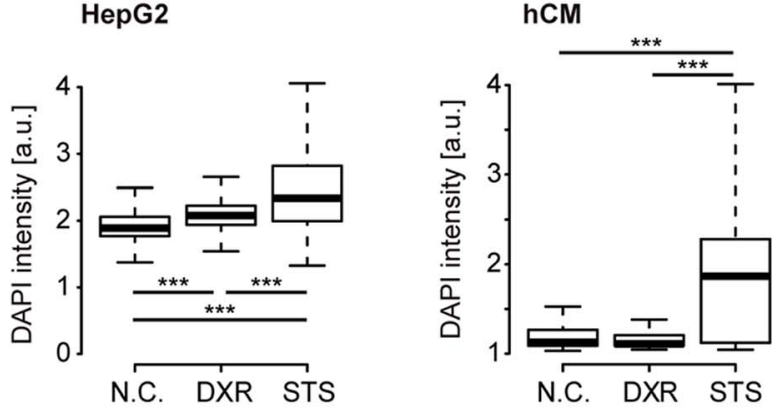

\section{C iHCC (Closed medium circulation)} HepG2
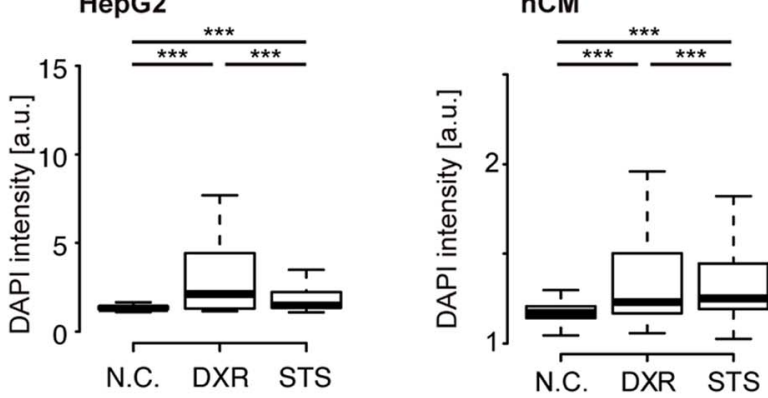

Fig. 7 iHCC serves as an experimental platform to model the side effects of the anti-cancer drug doxorubicin (DXR). (A) Fluorescent micrographs of hCMs treated with $20 \mu \mathrm{M}$ DXR in hCM medium for 24 hours in the iHCC. Staurosporine (STS; $1 \mu \mathrm{M}$ ) in hCM medium for 24 hours was used as a positive control for cell damage. DAPI staining was performed to visualize damaged cells. Untreated hCMs were used as the negative control (N.C.). Scale bar, $200 \mu \mathrm{m}$. (B, C) Quantitative single-cell profiling to reveal the effects of DXR on damaged HepG2 cells and hCMs cultured in a 96-well plate under static conditions (B) and in iHCC with closed medium circulation (C). DAPI fluorescence intensity in over 3000 individual cells was analyzed for each sample. PValues (*** < 0.001) were determined using Mann Whitney's $U$ test. The center lines show the medians; box limits indicate $25 \%$ and $75 \%$; whiskers extend 1.5 times the interquartile range from $25 \%$ and $75 \%$.

(Fig. 5B and C). These results suggest that the hCM medium allowed culture of both hCMs and HepG2 cells without damage during cell culture and treatment.

\section{Drug testing in iHCC}

Next, we performed iHCC-based assays to evaluate the efficacy and toxicity of DXR (Fig. 6). As a comparison, a conventional cell-based assay was also performed in a 96-well plate, in which only static conditions could be applied to the cells, and the interaction between hCMs and HepG2 cells via metabolites released from cells in cell culture medium could not be observed. As a positive control (P.C.) for cell damage, 

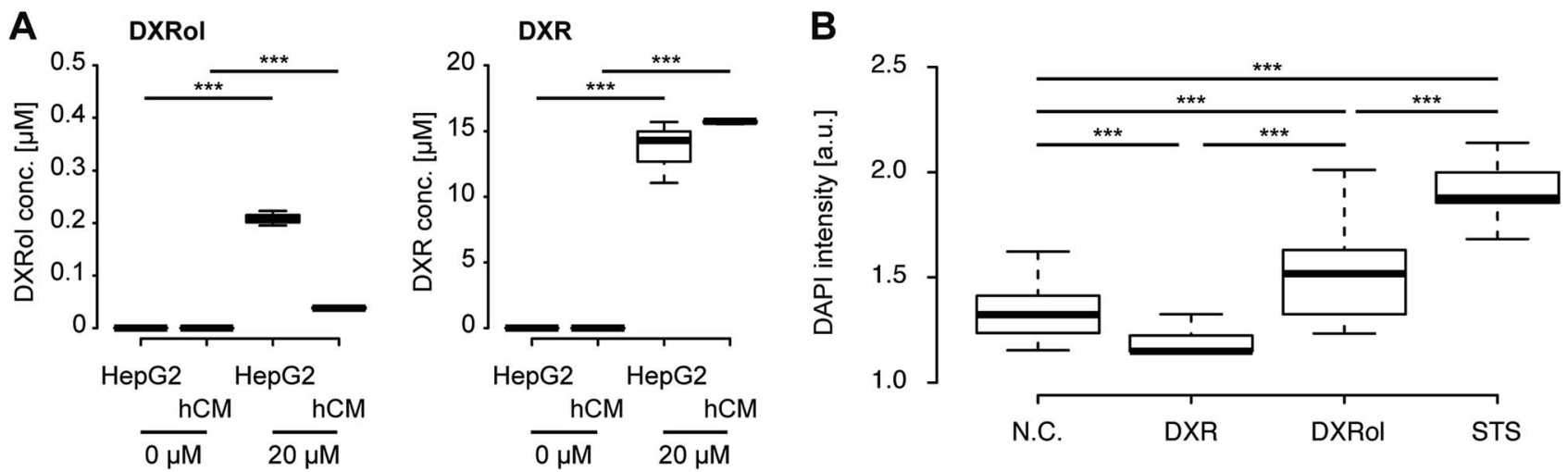

Fig. 8 Doxorubicinol (DXRol), but not doxorubicin (DXR), showed toxic effects on human cardiomyocytes (hCMs). (A) HepG2 cells metabolized DXR to DXRol, but hCMs did not (left panel). The DXR amount in the HepG2 culture was reduced compared with that in the hCM culture (right panel). HepG2 cells and hCMs were cultured in a 96-well plate and treated with DXR $(20 \mu M)$ for 24 hours. The culture medium supernatant was collected and analysed using ultra-high-performance liquid chromatography-mass spectrometry to measure both DXR and DXRol. (B) Quantitative single-cell profiling to investigate the effects of doxorubicin (DXR) and its metabolite, doxorubicinol (DXRol), on hCMs. hCMs were cultured in a 96-well plate and treated with DXR $(20 \mu \mathrm{M})$ or DXRol $(20 \mu \mathrm{M})$ for 24 hours. DAPI fluorescence intensity in over 200 individual cells was analyzed for each sample. P-Values $(* * *<0.001)$ were determined by Steel-Dwass test. The center lines show the medians; box limits indicate $25 \%$ and $75 \%$; whiskers extend 1.5 times the interquartile range from $25 \%$ and $75 \%$.

staurosporine (STS) ${ }^{43}$ was used to induce apoptosis. As a negative control (N.C.), a mock solution (hCM medium supplemented with $0.1 \%(\mathrm{v} / \mathrm{v})$ DMSO) was used. EdU was used as an indicator of cells in the S-phase, as DXR inhibits cell proliferation by intercalating into the genome during DNA duplication. In both experimental setups, HepG2 cells treated with $20 \mu \mathrm{M}$ DXR reduced EdU accumulation to levels comparable to those in cells treated with STS, resulting in lower cell proliferation (Fig. 6B). This result indicated that DXR showed anti-cancer effects on HepG2 cells. We also attempted EdU staining of hCMs, but since these were non-cancerous healthy cells and do not proliferate, no EdU signal was obtained, in contrast to HepG2 cells (Fig. S1 in ESI $\dagger$ ).

For better observation of DXR-induced cell damage, DAPI fluorescent dye was used as a dead cell assay (Fig. 7A). ${ }^{44,45}$ DAPI does not pass through the intact cell membrane, but penetrates the membrane of damaged or apoptotic cells and binds to the DNA. Therefore, DAPI fluorescence is only observed for dead or apoptotic cells. To conduct a cell-based assay in a microfluidic device, in situ methods such as cell imaging ${ }^{9}$ and the use of integrated sensors ${ }^{22}$ are more suitable than other methods involving cell lysis (e.g., enzyme-linked immune-sorbent assay, western blotting or quantitative polymerase chain reaction) or secretion (e.g., secreted alkaline phosphatase assay) because of the lack of sample loss in the device and tubing. To quantitatively analyze cell damage, single-cell profiling was also conducted using both iHCC and a parallel 96-well plate. Under static conditions in the 96-well plate, the number of cells damaged by DXR increased slightly, but only for HepG2 cells $\left({ }^{* * *} p<0.001\right.$, compared with N.C.), and not for the hCMs (Fig. 7B). This result indicated that DXR exerted an appropriate anti-cancer effect on HepG2 but did not cause cell damage to the hCMs. In contrast, the iHCC experiment produced different results (Fig. 7C). Following the application of closed-loop medium circulation with DXR in iHCC, the number of damaged hCMs increased significantly $\left({ }^{* * *} p<0.001\right.$, compared with NC), whereas no cell damage was observed without DXR. In light of these results, we hypothesized that the cytotoxicity was attributable to DXR metabolites being released from HepG2 cells, rather than to DXR itself, which then reached the hCM cells via the circulating medium.

DXRol (doxorubicinol), a known DXR metabolite, was used to validate our hypothesis. ${ }^{46,47}$ While the side effects of DXR on cardiomyocytes and its toxicity mechanism has been intensively studied, the effects of DXRol are not known. First, we measured the levels of DXRol produced from DXR-treated HepG2 and hCM cells (Fig. 8A left panel and Fig. S2 $\dagger$ ). HepG2 cells produced $0.20 \mu \mathrm{M}$ of DXRol in $100 \mu \mathrm{L}$ cell culture medium by metabolizing DXR, but hCMs did not. In fact, the amount of DXR in HepG2 culture was reduced compared with that in hCM culture due to its metabolism to DXRol (Fig. 8A right panel). To investigate the cytotoxic effects of DXR and DXRol, release of lactate dehydorogenase (LDH), a well-known indicator of cell damage, was determined in the 96-well plate assay (Fig. S3†). While $20 \mu \mathrm{M}$ DXR was cytotoxic to HepG2 cells, lower DXR concentrations did not show cytotoxicity. In terms of hCMs, DXR did not induce LDH release, indicating that DXR did not cause cell damage. In contrast, $20 \mu \mathrm{M}$ DXRol caused cytotoxic effects in hCMs, but not in HepG2 cells. As shown in Fig. 8A, HepG2 cells produced only $0.20 \mu \mathrm{M}$ DXRol from DXR, but this concentration did not cause cell damage to hCMs. This difference is largely due to the differences between the iHCC and 96well plate systems. In the case of the 96-well plate, metabolites released from cells might be diluted in the cell culture medium, whereas, in the case of iHCC, the metabolites were not diluted as much due to the smaller volume used for cell culture. Therefore, metabolites released from cells in the iHCC might more effectively affect other cells than they would affect the cells in a 96-well plate. In this regard, further analysis using a 96-well plate assay and $20 \mu \mathrm{M}$ of DXR and DXRol were used. To test the 
effects of DXRol on the proliferation of HepG2 cells, an EdU cell proliferation assay was carried out (Fig. S4 $\dagger$ ). As a result, DXRol significantly reduced the EdU staining intensity $(* * * P<0.001)$, indicating reduced cell proliferation; this result was similar to that obtained for DXR. Furthermore, hCMs treated with DXRol had more cell damage than hCMs treated with DXR or the N.C. $\left({ }^{* * *} P<0.001\right.$; Fig. $\left.8 \mathrm{~B}\right)$. These results suggest that DXR is not toxic to healthy cardiomyocytes, but its metabolite DXRol is very toxic to cardiomyocytes.

\section{Conclusions}

In conclusion, we propose a proof of concept for recapitulation of the cardiotoxic side effects of an anti-cancer drug in vitro using a simple iHCC, which comprises a microfluidic device allowing for integrated culture of cancer and heart cells. The iHCC developed here permits (1) culture of cells from different types of tissues within a single device, (2) application of a closed circulation loop as an artificial blood circulatory system, and (3) multiple sets of different samples to be used for systematic tests. Essentially, it allows a drug to travel through tissues via the circulation loop and to produce tissue-specific metabolites. Thus, the cytotoxicity of an anti-cancer drug on cancer cells and normal cardiomyocytes can be successfully evaluated within a device, thereby recapitulating the side effects. Because this cannot be achieved using conventional cell cultureware, iHCC offers great advantages over conventional OoC/BoC platforms to accurately reproduce human physiological conditions by effectively utilizing its circulation loop to connect different tissues. Furthermore, the proposed fabrication method for on-chip integration of micro pumps is scalable and applicable for other studies. Therefore, this is the first step in recapitulating the side effects of a drug using the $\mathrm{OoC} / \mathrm{BoC}$ platforms.

To make more advanced BoC platforms, there are several requirements. First, the use of more functional tissues for accurate drug testing is needed and this means that threedimensional cell culture needs to be performed in these BoC platforms. Of course, the microfluidic technique offers chemical and physical control over three-dimensional cellular environments. ${ }^{33,34,48-50}$ These advantages should allow for the generation of environments highly suitable for cells to express their functions, even in vitro.

Second, PDMS is known to absorb small molecules, such as hydrophobic molecules, ${ }^{19}$ which might influence the pharmacological results. ${ }^{51}$ Although alternatives to PDMS have been under development for a long time,${ }^{39}$ no suitable substitutes are yet available. Therefore, a new material enabling better pharmacological studies needs to be developed.

Third, it is also important to note that the cell culture medium needs to be optimized for the tested cell types, especially, for performing BoC experiments for longer periods, such as a month or more. In this study, we used the hCM medium for both cardiomyocytes and hepatocellular carcinoma cells. This issue can be addressed by both development of the cell culture medium and design of the microfluidic device.

Fourth, it is also important to note that the results obtained using the BoC platform need to be reconciled with pharmacological data obtained in either preclinical animal studies or clinical trials. Therefore, it is also necessary to design experiments and analyses to compare iHCC-based and preclinical/clinical trial data.

Finally, we envision that by applying the recently developed induced pluripotent stem cell technology and increasing the number and complexity of tissues using advanced microengineering technology, this platform will be able to recapitulate physiological and pathological conditions for precise investigation of drug efficacy and toxicity, which would be a significant step toward realizing personalized medicine.

\section{Acknowledgements}

We thank Ms Minako Nakajima and Dr Misao Takemoto for their assistance with cell culture, Dr Christopher Fockenberg for his assistance in the experimental setup and programming, Mr Kouta Kobayashi for device fabrication, and Ms Yumie Tokunaga for statistical analyses. We also thank the staff at Tokyo Ohka Kogyo Co. Ltd. for the helpful discussions. Funding was generously provided by the Japan Society for the Promotion of Science (JSPS; 24656502 and 16K14660 (KK)) and Terumo Life Science Foundation. The WPI-iCeMS is supported by the World Premier International Research Centre Initiative (WPI), the Ministry of Education, Culture, Sports, Science and Technology (MEXT), Japan. A part of this work was supported by the Nanotechnology Platform Project within MEXT, Japan, through Kyoto University Nano Technology Hub. The Medical Research Support Center, Graduate School of Medicine, Kyoto University was supported by the Platform of Drug Discovery, Informatics, and Structural Life Science from MEXT, Japan.

\section{Notes and references}

1 M. Abou-Gharbia and W. E. Childers, J. Med. Chem., 2014, 57, 5525-5553.

2 J. Zurlo, D. Rudacille and A. M. Goldberg, Animals and Alternatives in Testing: History, Science, and Ethics, Mary Ann Liebert, 1994.

3 S. N. Bhatia and D. E. Ingber, Nat. Biotechnol., 2014, 32, 760772.

4 M. B. Esch, J. M. Prot, Y. I. Wang, P. Miller, J. R. LlamasVidales, B. A. Naughton, D. R. Applegate and M. L. Shuler, Lab Chip, 2015, 15, 2269-2277.

5 S. Selimovic, M. R. Dokmeci and A. Khademhosseini, Curr. Opin. Pharmacol., 2013, 13, 829-833.

6 J. Fukumoto and N. Kolliputi, Front. Pharmacol., 2012, 3, 205.

7 C. T. Ho, R. Z. Lin, W. Y. Chang, H. Y. Chang and C. H. Liu, Lab Chip, 2006, 6, 724-734.

8 J. M. Prot, A. Bunescu, B. Elena-Herrmann, C. Aninat, L. C. Snouber, L. Griscom, F. Razan, F. Y. Bois, C. Legallais, C. Brochot, A. Corlu, M. E. Dumas and E. Leclerc, Toxicol. Appl. Pharmacol., 2012, 259, 270-280.

9 K. J. Jang, A. P. Mehr, G. A. Hamilton, L. A. McPartlin, S. Chung, K. Y. Suh and D. E. Ingber, Integr. Biol., 2013, 5, 1119-1129. 
10 H. J. Kim, D. Huh, G. Hamilton and D. E. Ingber, Lab Chip, 2012, 12, 2165-2174.

11 C. A. Dickey, C. Patterson, D. Dickson and L. Petrucelli, Trends Mol. Med., 2007, 13, 32-38.

12 A. Guan, P. Hamilton, Y. Wang, M. Gorbet, Z. Li and K. S. Phillips, Nature Biomedical Engineering, 2017, 1, 0045.

13 J. H. Sung, M. B. Esch, J. M. Prot, C. J. Long, A. Smith, J. J. Hickman and M. L. Shuler, Lab Chip, 2013, 13, 12011212.

14 H. E. Abaci and M. L. Shuler, Integr. Biol., 2015, 7, 383-391.

15 K. Kamei, Y. Hirai and O. Tabata, IEEE Nanotechnology Magazine, 2013, 7, 6-14.

16 M. B. Esch, T. L. King and M. L. Shuler, Annu. Rev. Biomed. Eng., 2011, 13, 55-72.

17 E.-M. Materne, A. P. Ramme, A. P. Terrasso, M. Serra, P. M. Alves, C. Brito, D. A. Sakharov, A. G. Tonevitsky, R. Lauster and U. Marx, J. Biotechnol., 2015, 205, 36-46.

18 J. H. Sung, C. Kam and M. L. Shuler, Lab Chip, 2010, 10, 446455.

19 P. M. Misun, J. Rothe, Y. R. F. Schmid, A. Hierlemann and O. Frey, Microsyst. Nanoeng., 2016, 2, 16022.

20 I. Maschmeyer, A. K. Lorenz, K. Schimek, T. Hasenberg, A. P. Ramme, J. Hubner, M. Lindner, C. Drewell, S. Bauer, A. Thomas, N. S. Sambo, F. Sonntag, R. Lauster and U. Marx, Lab Chip, 2015, 15, 2688-2699.

21 C. Oleaga, C. Bernabini, A. S. Smith, B. Srinivasan, M. Jackson, W. McLamb, V. Platt, R. Bridges, Y. Cai, N. Santhanam, B. Berry, S. Najjar, N. Akanda, X. Guo, C. Martin, G. Ekman, M. B. Esch, J. Langer, G. Ouedraogo, J. Cotovio, L. Breton, M. L. Shuler and J. J. Hickman, Sci. Rep., 2016, 6, 20030.

22 K. Hattori, Y. Munehira, H. Kobayashi, T. Satoh, S. Sugiura and T. Kanamori, J. Biosci. Bioeng., 2014, 118, 327-332.

23 M. Y. Rotenberg, E. Ruvinov, A. Armoza and S. Cohen, Lab Chip, 2012, 12, 2696.

24 M. A. Unger, H. P. Chou, T. Thorsen, A. Scherer and S. R. Quake, Science, 2000, 288, 113-116.

25 T. Thorsen, S. J. Maerkl and S. R. Quake, Science, 2002, 298, 580-584.

26 A. J. Dy, A. Cosmanescu, J. Sluka, J. A. Glazier, D. Stupack and D. Amarie, J. Micromech. Microeng., 2014, 24, 057001.

27 W. Park, S. Han and S. Kwon, Lab Chip, 2010, 10, 2814-2817. 28 S. S. Huang, Q. H. He, X. Q. Hu and H. W. Chen, J. Micromech. Microeng., 2012, 22, 085008.

29 A. Albini, G. Pennesi, F. Donatelli, R. Cammarota, S. De Flora and D. M. Noonan, J. Natl. Cancer Inst., 2010, 102, 14-25.

30 O. Tacar, P. Sriamornsak and C. R. Dass, J. Pharm. Pharmacol., 2013, 65, 157-170.
31 S. Yano, S. Li, Q. Han, Y. Tan, M. Bouvet, T. Fujiwara and R. M. Hoffman, Oncotarget, 2014, 5, 8729-8736.

32 K. Chatterjee, J. Zhang, N. Honbo and J. S. Karliner, Cardiology, 2010, 115, 155-162.

33 K. Kamei, Y. Hirai, M. Yoshioka, Y. Makino, Q. Yuan, M. Nakajima, Y. Chen and O. Tabata, Adv. Healthcare Mater., 2013, 2, 287-291.

34 K. Kamei, J. Lab. Autom., 2013, 18, 469-481.

35 Z. T. Yu, K. Kamei, H. Takahashi, C. J. Shu, X. Wang, G. W. He, R. Silverman, C. G. Radu, O. N. Witte, K. B. Lee and H. R. Tseng, Biomed. Microdevices, 2009, 11, 547-555.

36 H.-P. Chou, M. Unger and S. Quake, Biomed. Microdevices, 2001, 3, 323-330.

37 Y. N. Xia and G. M. Whitesides, Angew. Chem., Int. Ed., 1998, 37, 550-575.

38 X. Ma, Y. Kato, F. van Kempen, Y. Hirai, T. Tsuchiya, F. van Keulen and O. Tabata, J. Microelectromech. Syst., 2015, 24, 1856-1867.

39 Y. Kato, Y. Hirai, K. Kamei, T. Tsuchiya and O. Tabata, 18th International Conference on Solid-State Sensors, Actuators and Microsystems (TRANSDUCERS), 2015, pp. 1549-1552.

40 M. A. Eddings and B. K. Gale, J. Micromech. Microeng., 2006, 16, 2396-2402.

41 J. S. Go and S. Shoji, Sens. Actuators, A, 2004, 114, 438-444.

42 T. Satoh, G. Narazaki, R. Sugita, H. Kobayashi, S. Sugiura and T. Kanamori, Lab Chip, 2016, 16, 2339-2348.

43 C. A. Belmokhtar, J. Hillion and E. Segal-Bendirdjian, Oncogene, 2001, 20, 3354-3362.

44 M. Kubista, B. Akerman and B. Norden, Biochemistry, 1987, 26, 4545-4553.

45 P. Puustinen, A. Rytter, M. Mortensen, P. Kohonen, J. M. Moreira and M. Jaattela, J. Cell Biol., 2014, 204, 713727.

46 A. D. Hanna, A. Lam, S. Tham, A. F. Dulhunty and N. A. Beard, Mol. Pharmacol., 2014, 86, 438-449.

47 R. D. Olson, P. S. Mushlin, D. E. Brenner, S. Fleischer, B. J. Cusack, B. K. Chang and R. J. Boucek Jr, Proc. Natl. Acad. Sci. U. S. A., 1988, 85, 3585-3589.

48 S. Halldorsson, E. Lucumi, R. Gomez-Sjoberg and R. M. Fleming, Biosens. Bioelectron., 2015, 63, 218-231.

49 J. Avesar, T. B. Arye and S. Levenberg, Lab Chip, 2014, 14, 2161-2167.

50 M. Mehling and S. Tay, Curr. Opin. Biotechnol., 2014, 25, 95102.

51 E. W. Esch, A. Bahinski and D. Huh, Nat. Rev. Drug Discovery, 2015, 14, 248-260. 